\title{
COVID-19 caused hearing loss
}

\author{
Milisavljevic Dusan $^{1}\left[\right.$ D . Stankovic Milan ${ }^{1} \cdot$ Dordevic Nikola ${ }^{1}$
}

Received: 24 February 2021 / Accepted: 17 June 2021 / Published online: 8 July 2021

(c) The Author(s), under exclusive licence to Springer-Verlag GmbH Germany, part of Springer Nature 2021

\begin{abstract}
Purpose The objective of this cross-sectional study was to determine if there is a potential link between COVID-19 infection and hearing loss.

Methods The prospective study was conducted in the COVID Hospital Clinical Centre Niš, Serbia. We performed tonal audiometry and used a custom questionnaire and medical histories to determine the incidence of hearing loss in COVID-19 positive patients.

Results There were 74 patients with COVID-19 that met the inclusion criteria of this study and they composed our experimental group. Fifty-four (73\%) were men and 20 (27\%) women. There were $30(40.5 \%)$ patients with hearing loss. Seventeen patients had unilateral and 13 had bilateral hearing loss. Significant differences between hearing loss groups and control group were found across all age groups, but not at all frequencies. No important differences were found when unilateral hearing loss and bilateral hearing loss groups were compared. There were no significant differences in distributions of comorbidities between the patients with hearing loss and normal hearing patients.

Conclusions We found that 30 (40.5\%) of the COVID-19 positive patients had sensorineural type of hearing loss. Across all age groups, there were statistically significant differences in frequencies between the COVID-19 positive patients and the control group. There were no significant differences in distributions of comorbidities between the patients with hearing loss and normal hearing patients. Distribution of unilateral and bilateral hearing loss and audiogram types was also not significantly different between the age groups.
\end{abstract}

Keywords Hearing loss $\cdot$ Covid-19 $\cdot$ Audiometry

\section{Introduction}

The first case of pneumonia with unknown cause was reported to the WHO China Office on the 31st of December 2019 and in January 2020 WHO issued the first guidance on the novel coronavirus. Coronavirus Disease 2019 (COVID19) developed into pandemic and as of the 18th of October 2020 there were more than a 39 million confirmed cases with more than a 1 million confirmed deaths [1].

The most common symptoms of COVID-19 are fever, cough, sore throat, headache, muscle pain, diarrhea, dyspnea and taste and smell disturbances [2,3]. Coronavirus disease may be asymptomatic as well [4].

Milisavljevic Dusan

dusanorldusan@gmail.com

1 Department of Otolaryngology, University Clinic Centre Nis, Bul. Zorana Đinđića 48, Nis, Serbia
It is well known that viral infections can induce hearing loss, which can be congenital or acquired [5]. Viral infections are associated with sensorineural hearing loss (SNHL), but conductive or mixed hearing loss can also occur and it can be unilateral or bilateral [6].

There are many definitions of hearing loss. One of them uses audiometric criterion for SSNHL as a decrease in hearing of $\geq 30$ decibels affecting at least 3 consecutive frequencies. Whenever premorbid audiometry is unavailable, hearing loss is often defined in relation to the opposite ear's thresholds [7].

There are few reported cases of SNHL $[8,9]$ and one case of conductive hearing loss due to acute otitis media in COVID-19 [10]. Some authors suggest that hearing loss can be the only sign of this disease, since it was found in asymptomatic patient $[11,12]$. In addition, one case of complete deafness and profound SNHL on the opposite side and bilateral tinnitus as well was reported in patient who previously had intensive care treatment for COVID-19 [13]. 
The hearing loss in COVID-19 could also be the consequence of therapy with chloroquine and hydroxychloroquine, which are part of clinical practice guidelines for COVID-19 treatment in several countries [14]. These drugs are used for the treatment of malaria and some chronic inflammatory diseases as well. It is well known that these medications can induce hearing loss. Ototoxicity of chloroquine and hydroxychloroquine is the result of inner ear damage [15]. The recommended dose of these drugs for COVID-19 treatment is higher than for malaria; therefore, ototoxic effect could be higher [16].

\section{Objective}

The objective of this prospective cross-sectional study was to determine if there is a potential link between COVID-19 infection and hearing loss.

\section{Materials and methods}

This study was approved by the Ethics Committee of Clinical Centre Niš (protocol number 24722/7).

The study was conducted in the COVID Hospital Clinical Centre Niš, Serbia.

All patients were tested positive for SARS-CoV-19 virus using RT-PCR testing. Participants were chosen for this study if they had no prior history of hearing impairment, had not taken any ototoxic medications, had no history of hereditary hearing loss, and had normal otoscopic findings during the clinical exam.

We performed tonal audiometry and used a custom questionnaire to get additional information about their health status. Since audiometry is not a routinely performed in general population, anamnesis was the only mean to establish or exclude previous hearing loss. Every patient had their medical history taken twice, first time by the medical staff of the Covid hospital, and second time by the authors of this study. Only the patients who met upper mentioned criteria were selected to participate in the study.

Custom questionnaire helped us while taking anamnesis and to better understand their current medical status and the progression of the clinical picture. We attached the translated questionnaire as Appendix 1.

Tonal audiometry was done with the Interacoustics AD629 diagnostic audiometer and Radioear 3045 earphones. It was performed as a bedside procedure. Since there was no accurate way to determine the effect of other environmental conditions on health and mental status, we focused on adjusting for the environmental noise. To overcome the effect generated by the background noise, we used the correctional factor $(\mathrm{CF})$. The $\mathrm{CF}$ was calculated by finding the average differences in tonal audiometries at every frequency,

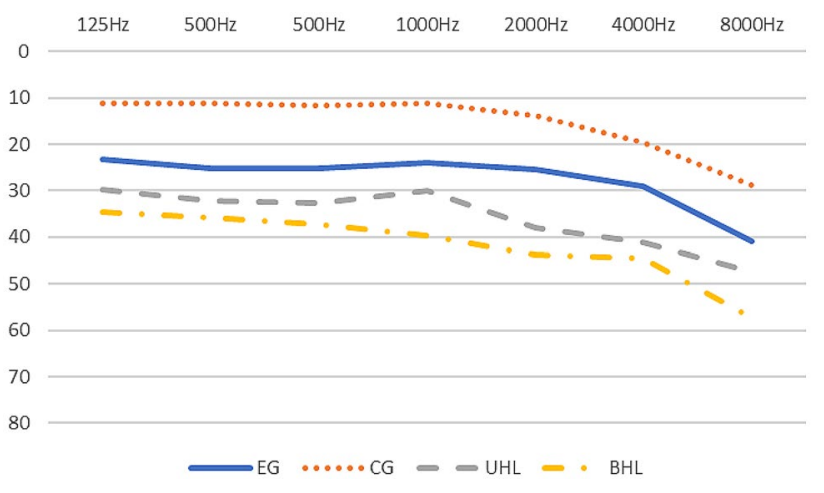

Fig. 1 Average audiograms of EG, CG, UHL and BHL groups. (EG experimental group, $C G$ control group, $U H L$ unilateral hearing loss, $B H L$ bilateral hearing loss)

performed in standard conditions and in the rooms that had the same conditions as ones in the COVID Hospital. Established $\mathrm{CF}$ was $15 \mathrm{~dB}$ at low and middle frequencies and $10 \mathrm{~dB}$ for $4000 \mathrm{~Hz}$ and $8000 \mathrm{~Hz}$.

Participants for the Control group were included through everyday outpatient praxis and were chosen if they weren't tested positive for SARS-CoV-19 virus using RT-PCR testing, had no prior history of hearing impairment, had not taken any ototoxic medications, had no history of hereditary hearing loss, and had normal otoscopic findings during the clinical exam. We used average findings at every frequency to construct the average audiogram of the Control group.

Patients tested in COVID Hospital were than compared with the ones in the Control group. We considered patients to have hearing loss if they had hearing impairment greater than the sum of the average hearing level and one standard deviation of the Control group at 3 or more frequencies. The ones with SNHL were placed in the hearing loss group (HL) and the rest in the no sensorineural hearing loss group (noHL). Patients with HL were then divided into those with unilateral hearing loss (UHL) and those with BHL (BHL).

In addition, the patients were divided in different groups regarding their gender, age and associated medical conditions. There were three different age groups: $\leq 55$ years, 56-65 years, and $>65$ years. All the medical conditions were divided into four subgroups: patients with no other medical condition, patients with arterial hypertension (HTA), patients with diabetes mellitus (DM) and patients with other medical conditions.

For the statistical analysis, we used IBM SPSS Statistics version 23. 


\section{Results}

The Control group (CG) consisted of 48 patients, 16 of them $(33 \%)$ were males and $32(67 \%)$ were females. There was no statistical difference at any frequency based on the gender. Mean age of the group was $60.83 \pm 11.32$ years (ages from 40 to 78 years). There was no statistical difference in the age between EG and CG. Average audiogram of the CG is shown in Fig. 1.

There were 74 patients that met the inclusion criteria of this study and they composed our experimental group (EG). Fifty-four $(73 \%)$ were men and $20(27 \%)$ women. There was no statistical difference at any frequency based on the gender, except at the $8000 \mathrm{~Hz}$, where women had significantly better hearing $(p<0.05)$. Mean age of the group was $60 \pm 10.85$ years (ages from 28 to 86 years). Mean age for men was 59.3 and for women 61.85 years. There was no when we compared all the patients with COVID-19 with the Control group.

Out of 74 patients that we examined, there were 30 (40.5\%) patients with hearing loss, which was sensorineural. Average values of hearing levels of EG, CG, UHL and BHL groups can be found in Table 1 .

Out of those 30 patients, seventeen patients had unilateral and 13 had bilateral hearing loss. Their audiograms were either flat ( $n=18,60 \%)$ or descending $(n=12,40 \%)$. None of the patients had ascending audiogram. This can be seen in Table 2. Distribution of unilateral and bilateral hearing loss and audiogram types did not significantly change between the age groups.

Out of all patients in HL group, 11 (36.7\%) were women and $19(63.3 \%)$ were men. We found that women had statistically significant greater hearing loss than men at 125 and $250 \mathrm{~Hz}(p<0.05)$ in patients with bilateral hearing loss. No

Table 1 Average values of hearing levels in $\mathrm{dB}$ for groups EG, CG, UHL and BHL (results represent average findings for both ears, except in the UHL group, where average values of the affected ear are represented)

\begin{tabular}{lllllllll}
\hline Groups & $n$ & $125 \mathrm{~Hz}$ & $500 \mathrm{~Hz}$ & $500 \mathrm{~Hz}$ & $1000 \mathrm{~Hz}$ & $2000 \mathrm{~Hz}$ & $4000 \mathrm{~Hz}$ & $8000 \mathrm{~Hz}$ \\
\hline EG & 74 & 23.31 & 25.07 & 25.27 & 24.05 & 25.47 & 28.92 & 40.95 \\
CG & 48 & 11.25 & 11.25 & 11.67 & 11.25 & 13.75 & 19.58 \\
UHL & 17 & 29.7 & 32.06 & 32.65 & 30 & 37.94 & 41.18 & 47.35 \\
BHL & 13 & 34.62 & 35.77 & 37.31 & 39.62 & 43.85 & 44.62 & 57.31 \\
\hline
\end{tabular}

$E G$ experimental group, $C G$ control group, $U H L$ unilateral hearing loss, $B H L$ bilateral hearing loss

Table 2 Patients with HL represented according to the type of hearing loss and audiogram type

\begin{tabular}{|c|c|c|c|c|}
\hline \multicolumn{2}{|c|}{ Age } & \multirow{2}{*}{$\begin{array}{l}\text { Unilateral } \\
14\end{array}$} & \multirow{2}{*}{$\frac{\text { Bilateral }}{4}$} & \multirow{2}{*}{$\frac{\text { Total }}{18}$} \\
\hline Flat & Total & & & \\
\hline & $\leq 55$ & 2 & 1 & 3 \\
\hline & $55-65$ & 7 & 3 & 10 \\
\hline & $>65$ & 5 & 0 & 5 \\
\hline \multirow[t]{4}{*}{ Descendent } & Total & 3 & 9 & 12 \\
\hline & $\leq 55$ & 0 & 2 & 2 \\
\hline & $55-65$ & 1 & 4 & 5 \\
\hline & $>65$ & 2 & 3 & 5 \\
\hline \multirow[t]{4}{*}{ Total } & Total & 17 & 13 & 30 \\
\hline & $\leq 55$ & 2 & 3 & 5 \\
\hline & $55-65$ & 8 & 7 & 15 \\
\hline & $>65$ & 7 & 3 & 10 \\
\hline
\end{tabular}

Flat $=$ flat type audiogram, Descendent $=$ descendent type of audiogram, Unilateral $=$ unilateral hearing loss, Bilateral=bilateral hearing loss

statistical difference in age between men and women. After applying the $\mathrm{CF}$ to the hearing levels of the $\mathrm{EG}$, we got the average audiogram of the EG, represented in Fig. 1. We found statistically significant difference at every frequency other differences regarding gender were found.

Average audiograms of the HL and CG groups divided into three different age groups are shown in Figs. 2 and 3 


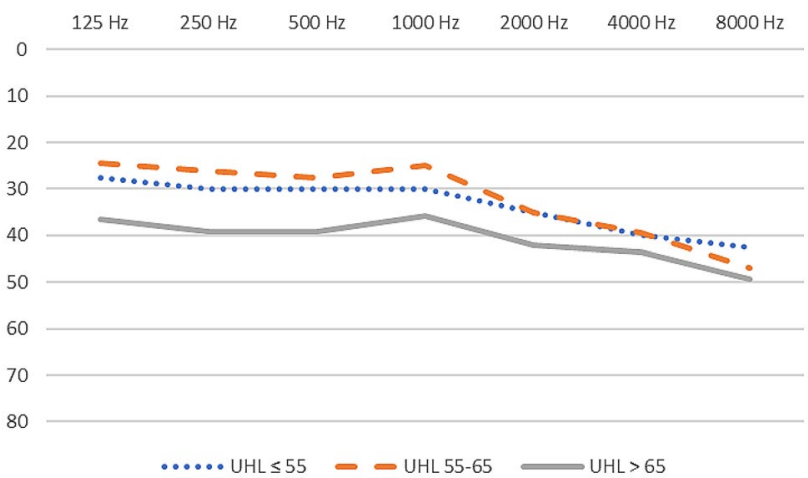

Fig. 2 Average audiograms of the UHL group, by the age groups. (UHL unilateral hearing loss)

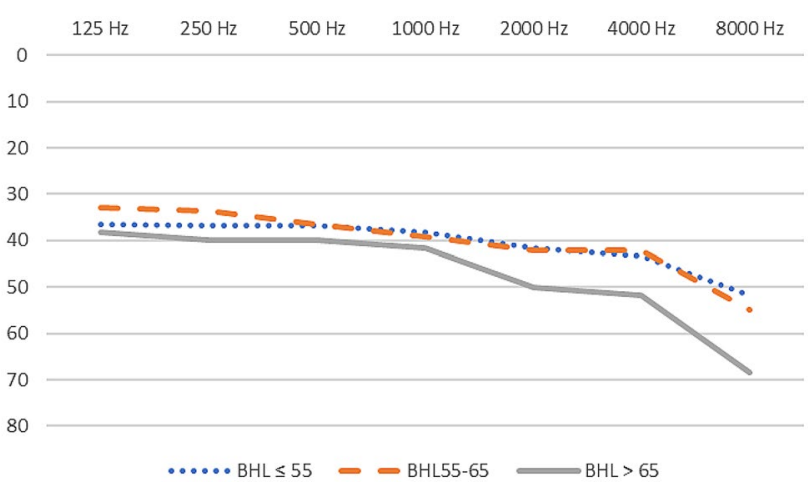

Fig. 3 Average audiograms of the BHL group, by the age groups. (BHL bilateral hearing loss)
$4000 \mathrm{~Hz}$ when compared to the patients that were younger than 55 years. Statistically significant differences were found when UHL, BHL and CG groups were compared by the age groups. In patients with the age up to 55 years, there were differences at every frequency when $\mathrm{CG}$ group was compared with BHL and only at 2000 and $4000 \mathrm{~Hz}$ when compared to the UHL. There was no difference between UHL and BHL in this age group. When comparing ages of 55-65 years, hearing impairment was statistically higher in UHL and BHL groups than that in CG at every frequency, except at $125 \mathrm{~Hz}$ when comparing UHL and CG. Differences between UHL and BHL were found only at $1000 \mathrm{~Hz}$, where BHL had greater hearing impairment. Patients that had UHL and were older than 65 years had statistically significant differences at $125-2000 \mathrm{~Hz}$ when compared to CG. Patients with BHL had greater hearing loss at every frequency compared to CG, except at $4000 \mathrm{~Hz}$, where $p$ was equal to 0.051 . There were no differences between UHL and BHL groups in patients in the oldest age group.

Out of all patients, $46(62.2 \%)$ patients had HTA, 16 (21.6\%) had DM, and 30 (40.5\%) of them had other medical conditions besides COVID-19 infection (Table 4). There were no significant differences in distributions of comorbidities between the patients with hearing loss and normal hearing patients. HTA, DM and other medical conditions did not have a significant impact on the level of hearing loss in patients with impaired hearing. However, patients that had normal hearing had statistically worse hearing level at 125 , 500,4000 and $8000 \mathrm{~Hz}$ if they also had HTA and at 4000 and $8000 \mathrm{~Hz}$ if they also had DM.

Patients with descending audiograms had statistically

Table 3 Average values of hearing levels in $\mathrm{dB}$ for patients in different age groups

\begin{tabular}{llllllll}
\hline Groups & $125 \mathrm{~Hz}$ & $500 \mathrm{~Hz}$ & $500 \mathrm{~Hz}$ & $1000 \mathrm{~Hz}$ & $2000 \mathrm{~Hz}$ & $4000 \mathrm{~Hz}$ & $8000 \mathrm{~Hz}$ \\
\hline UHL $\leq 55$ & $27.5 \pm 17.68$ & $30 \pm 14.14$ & $30 \pm 14.14$ & $30 \pm 14.14$ & $35 \pm 14.14$ & $40 \pm 7.07$ & $42.5 \pm 24.75$ \\
UHL 55-65 & $24.375 \pm 11.16$ & $26.25 \pm 7.91$ & $27.5 \pm 9.26$ & $25 \pm 7.07$ & $35 \pm 9.64$ & $39.375 \pm 12.08$ & $46.875 \pm 11.93$ \\
UHL $>$ 65 & $36.43 \pm 9.88$ & $39.29 \pm 11.34$ & $39.29 \pm 8.86$ & $35.71 \pm 10.18$ & $42.14 \pm 18.86$ & $43.57 \pm 10.29$ & $49.29 \pm 11.7$ \\
BHL $\leq 55$ & $35 \pm 5$ & $36.67 \pm 7.64$ & $36.67 \pm 7.64$ & $38.33 \pm 10.41$ & $41.67 \pm 7.64$ & $43.33 \pm 2.89$ & $51.67 \pm 7.64$ \\
BHL 55-65 & $32.86 \pm 10.75$ & $33.57 \pm 12.15$ & $36.43 \pm 11.44$ & $39.29 \pm 14.84$ & $42.14 \pm 14.1$ & $42.14 \pm 8.59$ & $55 \pm 16.07$ \\
BHL $>$ 65 & $38.33 \pm 2.89$ & $40 \pm 0$ & $40 \pm 0$ & $41.67 \pm 5.7735$ & $50 \pm 5$ & $51.67 \pm 2.89$ & $68.33 \pm 14.43$ \\
CG $\leq 55$ & $10 \pm 10$ & $10 \pm 10$ & $10 \pm 10$ & $3.33 \pm 5.77$ & $5 \pm 5$ & $5 \pm 5$ & $13.33 \pm 5.77$ \\
CG 55-65 & $13 \pm 10.95$ & $12 \pm 7.58$ & $13 \pm 8.36$ & $15 \pm 8.66$ & $15 \pm 10$ & $20 \pm 12.74$ & $23 \pm 11.51$ \\
CG $>$ 65 & $10 \pm 8.16$ & $11.25 \pm 8.53$ & $11.25 \pm 8.53$ & $11.25 \pm 12.58$ & $18.75 \pm 14.36$ & $30 \pm 14.14$ & $47.5 \pm 17.07$ \\
\hline
\end{tabular}

$U H L$ unilateral hearing loss, $B H L$ bilateral hearing loss, $C G$ control group

and values of the audiograms for this groups are shown in Table 3.

No significant differences were found when comparing UHL by age groups and BHL by age groups. Patients in the CG group over the age of 65 years had significantly higher thresholds at $8000 \mathrm{~Hz}$ then both of other age groups and at greater hearing loss at 2000, 4000 and $8000 \mathrm{~Hz}$ compared with those with flat audiograms. 
Table 4 Hearing loss and comorbidities in COVID-19 patients

\begin{tabular}{llllll}
\hline Comorbidity & $\begin{array}{l}\text { Unilateral } \\
(17)\end{array}$ & $\begin{array}{l}\text { Bilateral } \\
(13)\end{array}$ & $\begin{array}{l}\text { Normal } \\
\text { hearing } \\
(44)\end{array}$ & Total (74) \\
\hline HTA & With & 13 & 9 & 24 & 46 \\
& Without & 4 & 4 & 20 & 28 \\
DM & With & 6 & 3 & 7 & 16 \\
& Without & 11 & 10 & 37 & 58 \\
\multirow{2}{*}{ Other } & With & 10 & 6 & 14 & 30 \\
& Without & 7 & 7 & 30 & 47 \\
\hline
\end{tabular}

HTA $=$ arterial hypertension, $\mathrm{DM}=$ diabetes mellitus, other $=$ other comorbidities not including arterial hypertension and diabetes mellitus, Unilateral = unilateral hearing loss, Bilateral=bilateral hearing loss

\section{Discussion}

Outbreak of the SARS-COVID-19 infection generated plenty of new emerging studies. Many of them have shown that COVID-19 can lead to a large spectrum of ENT symptoms. Some of them include sore throat, hyposmia/anosmia, hypogeusia/ageusia, dysphagia, tinnitus, vertigo and hearing loss. However, only a small number of these address the otologic aspect of this disease. Since COVID-19 clearly affects olfactory and gustatory functions [17], it is not farfetched that it can also have an impact on the auditory system. Osman Kilic et al. were among the first authors that indicated that there may be a link between COVID-19 and SNHL [11].

The difficulties in acquiring adequate audiometry in bedside conditions are already mentioned in literature $[18,19]$.

In our study, women had significantly better thresholds at $8000 \mathrm{~Hz}$ in the EG group. However, in the BHL group, men had significantly better hearing at 125 and $250 \mathrm{~Hz}$, but no differences at other frequencies. There were no significant differences between genders in the CG. This type of disparity between genders (men performed better at lower frequencies and women at higher frequencies across all age groups) has been previously described in the literature in large cohort studies, the most recent one conducted by Homans $\mathrm{CN}$ et al. [20].

Out of all patients in the EG, in thirty (40.5\%) of them we found signs of hearing loss. This relatively high percentage may be caused by the type of patients that were included in this study. We examined only the patients with moderate clinical presentations. Asymptomatic and mild cases were not treated in the hospital and severe and critical cases were not suitable candidates for tonal audiometry. We do not have a clear understanding how investigated and found hearing loss in this group of patients is portrayed in general population. We found both unilateral hearing loss, which is typical for sudden SNHL and bilateral hearing loss, which is also possible in SNHL [21]. Rhaman AS and Wahid AA reported a case of COVID-19 positive patient that did not present with typical symptoms, but complained of sudden onset of the unilateral hearing loss and tinnitus [22]. On the other hand, Degen C et al. reported a case of a bilateral acute profound SNHL after COVID-19 pneumonia [13].

Audiograms were either flat $(n=18,60 \%)$ or descending $(n=12,40 \%)$ type. The fact that some of the patients had worse hearing thresholds at lower frequencies than CG can be attributed to the potential changes in the middle ear. It is also mentioned in the literature that COVID-19 can induce acute otitis media in adults [10].

In our study, statistically significant difference at every frequency $(p<0.001)$ was found when UHL and BHL groups were compared with the CG group. Mustafa MWM has shown that there is a significant difference at higher frequencies between asymptomatic COVID-19 infected patients and control group, audiometric thresholds being higher in the test group [12].The precise reason for hearing loss is currently unknown, but there are a lot of mechanisms by which virus could affect auditory system. Recent studies have indicated the importance of endothelial dysfunction and micro thrombosis in COVID-19 infection [23]. Autopsies performed in Hamburg on 12 patients that died with confirmed diagnosis of COVID-19 infection confirmed high incidence of thromboembolic events in these patients [24]. It is shown that virus can affect many organs, by binding to the ACE2 receptor, including cochlea, cochlear nerve and central nervous system [25]. The virus is associated with the increased activation of the immune system that can damage the patient's tissue [26, 27]. Difference in the lower frequencies could potentially be explained by the spreading of the infection from the nasopharynx that may lead to effusion of the middle ear $[10,28]$.

When comparing patients in HL groups to each other according to the age, there was increase in audiometric thresholds in older groups, as expected, but had no statistical significance possibly due to the small number of patients. Patients in the CG group over the age of 65 years had significantly higher thresholds at $8000 \mathrm{~Hz}$ then both of other age groups and at $4000 \mathrm{~Hz}$ when compared to the patients that were younger than 55 years. This can be explained by the common age-related hearing loss that happens predominately at higher frequencies [20].

Significant differences between HL groups and CG group were found across all age groups, but not at all frequencies. Small or no differences were found when UHL and BHL groups were compared. Greater differences between the groups may be present, but small number of patients per groups preclude any statistical significance.

Patients that had normal hearing had statistically worse hearing level at 125, 500, 4000 and $8000 \mathrm{~Hz}$ if they also had HTA and at 4000 and $8000 \mathrm{~Hz}$ if they also had DM. This effect of HTA and DM is well documented by the other 
authors [29]. The fact that we did not find any significant differences when comparing comorbidities in patients with hearing loss may be caused by the small group sizes.

Further research of this topic is needed to procure a better understanding of the effect that SARS-COVID-19 has on auditory system, as well as potential long-term risks and treatment options. With that in mind, we plan on performing a follow up study of these patients, their treatment and outcome.

\section{Limitations of the study}

Limitations of this study include lack of standardised testing environment for tonal audiometry and relatively low number of examined patients. We conducted this research only on patients with moderate COVID-19 cases.

\section{Conclusion}

We found that $30(40.5 \%)$ of the COVID-19 positive patients had sensorineural type of hearing loss. Across all age groups, there were statistically significant differences in frequencies between the COVID-19 positive patients and the control group. There were no significant differences in distributions of comorbidities between the patients with hearing loss and normal hearing patients. Distribution of unilateral and bilateral hearing loss and audiogram types was also not significantly different between the age groups.

\section{Appendix 1}

Translated custom questionnaire used for procuring additional information about the patient's health status 
1. Name and surname

2. Sex Male

Female

3. Age

4. Phone number

5. Address

6. Smoking habits

Doesn't smoke

Used to smoke

Smokes

7. Chronic diseases

8. Medications regularly taken

9. Date of Covid-19 virus detection

10. Date of virus exposure (if known)

11. Date of the first symptoms

12. What were the first symptoms?

- cough

- $\quad$ sore throat

- fatigue

- fever

- muscle pain
- nasal secretion

- nasal

obstruction

- smell disorder

- $\quad$ taste disorder

- headache

13. Family history of hereditary hearing loss

Yes No

14. Noise exposure during lifetime

Yes

No

15. Earlier hearing problems

Yes

No

16. Current therapy in Covid hospital

17. Presence of the following symptoms: deafness, tinnitus, instability, dizziness?

18. Changes in the detection of spicy food?

Yes

No

19. Changes in the detection of salty food?

Yes

No

20. Changes in the detection of sour food?

Yes

No

21. Changes in the detection of sweet food?

Yes

No 
22. Changes in the sense of taste prior to Covid-19 related symptoms? Yes No

23. Usual sense of taste strength on the scale from 0 to 10 ?

24. Current sense of taste strength on the scale from 0 to 10 ?

25. Changes in the sense of smell prior to Covid-19 related symptoms? Yes No

26. Changes in the sense of smell during Covid-19 related symptoms? Yes No

27. If there is a problem, when did it start:

- on the first day

- between the second and fifth day

- after fifth day

28. Usual sense of smell strength on the scale from 0 to 10 ?

29. Current sense of smell strength on the scale from 0 to 10 ?

30. ENT clinical findings

31. Tonal audiometry findings

D

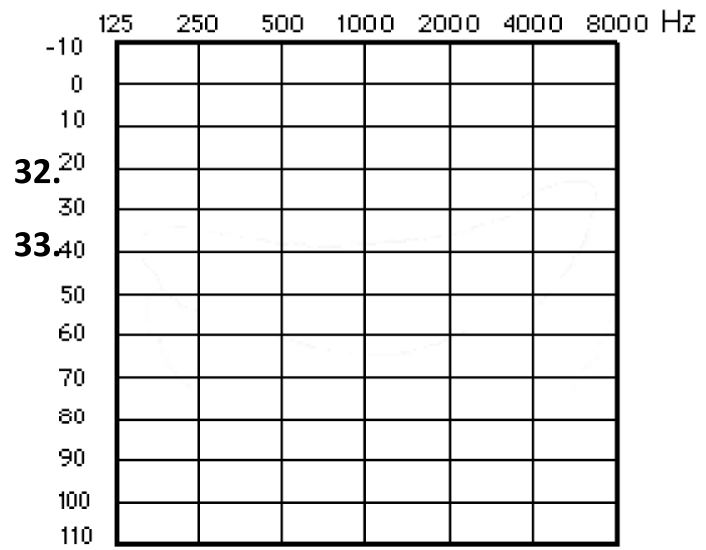

$\mathrm{S}$

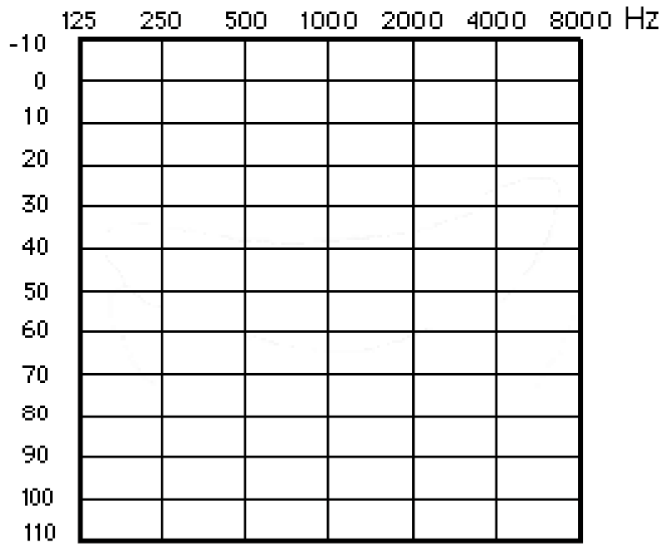

34. Laboratory findings

35. Other comments 
Author contributions All authors contributed to this work. The study was designed by prof. MD and prof. SM. Data collection and analysis were performed by DN. The first draft of the manuscript was written by all authors. Reviewing and editing was performed by prof. MD and prof. SM.

Funding No funding was received to assist with the preparation of this manuscript.

Data availability Availability of data and materials is made available on request.

\section{Declarations}

Conflict of interest The authors have no relevant financial or non-financial interests to disclose.

Ethics approval This study was performed in line with the principles of the Declaration of Helsinki. Approval was granted by the Ethics Committee of Clinical Centre Niš, Serbia (protocol number 24722/7).

Consent to participate Informed consent was obtained from all individual participants included in the study.

Consent to publish Informed consent was obtained from all individual participants included in the study.

\section{References}

1. Coronavirus disease (COVID-19)—World Health Organization (2020) https://www.who.int/emergencies/diseases/novel-coron avirus-2019. Acessed 18 Oct 2020

2. Cao Y, Liu X, Xiong L, Cai K (2020) Imaging and clinical features of patients with 2019 novel coronavirus SARS-CoV-2: a systematic review and meta-analysis. J Med Virol, https://www. ncbi.nlm.nih.gov/pmc/articles/PMC7228215/. Acessed 18 Oct 2020

3. Freni F, Meduri A, Gazia F, Nicastro V, Galletti C, Aragona P et al (2020) Symptomatology in head and neck district in coronavirus disease (COVID-19): a possible neuroinvasive action of SARSCoV-2. Am J Otolaryngol 41(5):102612. https://doi.org/10.1016/j. amjoto.2020.102612

4. Kim G-U, Kim M-J, Ra SH, Lee J, Bae S, Jung J et al (2020) Clinical characteristics of asymptomatic and symptomatic patients with mild COVID-19. Clin Microbiol Infect 26(7):948.e1-948.e3. https://doi.org/10.1016/j.cmi.2020.04.040

5. Cohen BE, Durstenfeld A, Roehm PC (2014) Viral causes of hearing loss: a review for hearing health professionals. Trends Hear 18:233121651454136. https://doi.org/10.1177/2331216514 541361

6. Young Y-H (2020) Contemporary review of the causes and differential diagnosis of sudden sensorineural hearing loss. Int J Audiol 59(4):243-253. https://doi.org/10.1080/14992027.2019.1689432

7. Chandrasekhar SS, Tsai Do BS, Schwartz SR, Bontempo LJ, Faucett EA, Finestone SA et al (2019) Clinical practice guideline: sudden hearing loss (update). Otolaryngol Head Neck Surg 161(1_suppl):S1-S45. https://doi.org/10.1177/0194599819 859885

8. Kalcioglu MT, Cag Y, Kilic O, Tuysuz O (2020) May COVID-19 cause sudden sensorineural hearing loss? Int J Infect Dis, https:// www.ijidonline.com/article/S1201-9712(20)32184-6/abstract. Acessed 18 Oct 2020
9. Elibol E (2021) Otolaryngological symptoms in COVID-19. Eur Arch Otorhinolaryngol 278(4):1233-1236. https://doi.org/10. 1007/s00405-020-06319-7

10. Fidan V (2020) New type of corona virus induced acute otitis media in adult. Am J Otolaryngol, https://www.ncbi.nlm.nih.gov/ pmc/articles/PMC7161479/. Acessed 18 Oct 2020

11. Kilic O, Kalcioglu MT, Cag Y, Tuysuz O, Pektas E, Caskurlu H et al (2020) Could sudden sensorineural hearing loss be the sole manifestation of COVID-19? An investigation into SARS-COV-2 in the etiology of sudden sensorineural hearing loss. Int J Infect Dis 97:208-211. https://doi.org/10.1016/j.ijid.2020.06.023

12. Mustafa MWM (2020) Audiological profile of asymptomatic Covid-19 PCR-positive cases. Am J Otolaryngol 41(3):102483. https://doi.org/10.1016/j.amjoto.2020.102483

13. Degen C, Lenarz T, Willenborg K (2020) Acute profound sensorineural hearing loss after COVID-19 pneumonia. Mayo Clin Proc 95(8):1801-1803. https://doi.org/10.1016/j.mayocp.2020.05.034

14. Jie Z, Hu HH, Zhi XZ (2020) The multicenter collaboration group of Department of Science and Technology of Guangdong Province and Health Commission of Guangdong Province for chloroquine in the treatment of novel coronavirus pneumonia. Expert consensus on chloroquine phosphate for the treatment of novel coronavirus pneumonia. Chinese 43(3):185-188. https://doi.org/ 10.3760/cma.j.issn.1001-0939.2020.03.009

15. Cianfrone G, Pentangelo D, Cianfrone F, Mazzei F, Turchetta R, Orlando MP et al (2011) Pharmacological drugs inducing ototoxicity, vestibular symptoms and tinnitus: a reasoned and updated guide. Eur Rev Med Pharmacol Sci 15(6):601-636

16. Prayuenyong P, Kasbekar AV, Baguley DM (2020) Clinical implications of chloroquine and hydroxychloroquine ototoxicity for COVID-19 treatment: a mini-review. Front Public Health 8:252. https://doi.org/10.3389/fpubh.2020.00252

17. Sakalli E, Temirbekov D, Bayri E, Alis EE, Erdurak SC, Bayraktaroglu M (2020) Ear nose throat-related symptoms with a focus on loss of smell and/or taste in COVID-19 patients. Am J Otolaryngol 41(6):102622. https://doi.org/10.1016/j.amjoto.2020. 102622 (published online ahead of print, 2020 Jun 23)

18. De Sousa KC, Smits C, Moore DR, Myburgh HC, Swanepoel DW (2020) Pure-tone audiometry without bone-conduction thresholds: using the digits-in-noise test to detect conductive hearing loss. Int J Audiol 59(10):801-808. https://doi.org/10.1080/14992027.2020. 1783585

19. Convery E, Keidser G, Seeto M, Freeston K, Zhou D, Dillon H (2014) Identification of conductive hearing loss using air conduction tests alone: reliability and validity of an automatic test battery. Ear Hear 35(1):e1-e8. https://doi.org/10.1097/AUD.0b013 e31829e $058 \mathrm{f}$

20. Homans NC, Metselaar RM, Dingemanse JG et al (2017) Prevalence of age-related hearing loss, including sex differences, in older adults in a large cohort study. Laryngoscope 127(3):725730. https://doi.org/10.1002/lary.26150

21. Lazarini PR, Camargo AC (2006) Idiopathic sudden sensorineural hearing loss: etiopathogenic aspects. Braz J Otorhinolaryngol 72(4):554-561. https://doi.org/10.1016/s1808-8694(15)31004-1

22. Abdel Rhman S, Abdel Wahid A (2020) COVID-19 and sudden sensorineural hearingloss, a case report. Otolaryngol Case Rep 16:100198

23. McFadyen JD, Stevens H, Peter K (2020) The emerging threat of (micro)thrombosis in COVID-19 and Its therapeutic implications. Circ Res 127(4):571-587

24. Wichmann D, Sperhake JP, Lütgehetmann M, Steurer S, Edler C, Heinemann A et al (2020) Autopsy findings and venous thromboembolism in patients with COVID-19: a prospective cohort study. Ann Intern Med 173(4):268-277. https://doi.org/10.7326/ M20-2003 
25. Harenberg J, Jonas JB, Trecca EMC (2020) A liaison between sudden sensorineural hearing loss and SARS-CoV-2 infection. Thromb Haemost 120(9):1237-1239. https://doi.org/10.1055/s0040-1714370

26. Delgado-Roche L, Mesta F (2020) Oxidative stress as key player in severe acute respiratory syndrome coronavirus (SARS-CoV) infection. Arch Med Res 51(5):384-387. https://doi.org/10.1016/j. arcmed.2020.04.019

27. Nile SH, Nile A, Qiu J, Li L, Jia X, Kai G (2020) COVID-19: pathogenesis, cytokine storm and therapeutic potential of interferons. Cytokine Growth Factor Rev 53:66-70. https://doi.org/10. 1016/j.cytogfr.2020.05.002
28. Saniasiaya J (2020) Hearing loss in SARS-CoV-2: what do we know? Ear, Nose Throat J. https://doi.org/10.1177/0145561320 946902

29. Meneses-Barriviera CL, Bazoni JA, Doi MY, Marchiori LLM (2018) Probable association of hearing loss, hypertension and diabetes mellitus in the elderly. Int Arch Otorhinolaryngol 22(4):337-341. https://doi.org/10.1055/s-0037-1606644

Publisher's Note Springer Nature remains neutral with regard to jurisdictional claims in published maps and institutional affiliations. 